\title{
WSN Architecture Design Based on Software Defined Networks
}

\author{
Tian He \\ Engineering Practice Center \\ Liaoning institute of Science and Technology \\ Liaoning Benxi, China \\ tianher@sina.cn
}

\author{
Zhao Hai \\ School of Computer Science and Engineering \\ Northeastern University \\ Shenyang, China \\ zhaohai@ise.neu.edu.cn
}

\author{
Shao Shi-liang \\ Robotics Laboratory \\ Shenyang Institute of Automation Chinese Academy of Sciences \\ Shenyang, China \\ shaoshiliangswu@163.com
}

\begin{abstract}
In order to improve the performance of wireless sensor networks and adapt the development of network technology, proposed a software defined wireless sensor network model which had hierarchy architecture and based on OpenFlow protocol under the software defined network architecture. Added the information fusion layer to the model, the Sink-nodes forwarded the link information after the sensor-nodes had collected information. Fused and fragmented logically the node data information using network virtualization technology of the OpenFlow protocol. Managed centrally the path forward strategy by the controller, and then improved the network information forward mechanism under the new architecture. On that basis, simulated the new architecture model and contrasted traditional wireless sensor routing protocols. The experiment results show that the new architecture can extend the life cycle of network and improve network.
\end{abstract}

Keywords-wireless sensor networks; software defined networks; network architecture; OpenFlow

\section{INTRODUCTION}

Wireless sensor networks (WSN) is a self-organizing network composed of a set of sensor nodes [1]. The nodes perceive and collect the sensing objects' information in the monitoring area, and then transfer them to the user. However, the development and application of traditional network architecture are more and more difficult with the rapid development of computers and Internet technology. Traditional TCP/IP protocol in the new form of network environment can not give full play to its advantages, which restricts the development for computer network technology. On the other hand, the hardware and information distribution strategy in WSN based on the traditional network architecture are all solidified. If the network bursts, the routing strategy will not change correspondingly.

Software defined networks (SDN) technology makes the control plane and the data plane decoupled, so that the control

Project supported by the National Natural Science Foundation of China (60973022, 61373159), and the national high-tech research and development plan (863 plan) (2013 AA102505). function is centralized to the central controller [2] and data forwarding is placed on a switch that supports OpenFlow, which makes the interaction between the application and network service and devices integrated tightly. The development and design of OpenFlow enable scholars to experiment and innovate to new protocols on a daily network. Furthermore, introducing SDN technology into WSN can simplify the hardware structure [3], improve the routing efficiency and optimize the resource allocation.

This article introduces OpenFlow/SDN technology into WSN to reform and reconstruct the traditional WSN architecture. According to the network virtualization technology application in SDN, add letter melt layer in the new architecture to make information transfer, fusion and control decoupling, then propose a kind of software defined wireless sensor network with information fusion (SD-WSN) and simulate the life cycle and transmission efficiency of the new architecture model. Compared with the traditional routing protocol, the proposed architecture model has obviously improvement in transmission efficiency and life cycle.

\section{CORRELATION THEORIES}

\section{A. WSN hierarchical Architecture}

Nodes in WSN are divided into management nodes, sensor nodes and sink nodes according to the execution type. Sensor nodes which can collect data are scattered in the specified sensing area. They cooperate with each other to complete information acquisition, storage and delivery and mutual transfer data information to sink nodes by multi-hop routing [4]. Finally, data in the whole area is transmitted to the remote control center in Internet (communication satellite) through the sink links [5].

Base on the OSI model and TCP/IP protocol cluster structure in traditional computer network, WSN also has Hierarchical Architecture as shown in fig.1. 


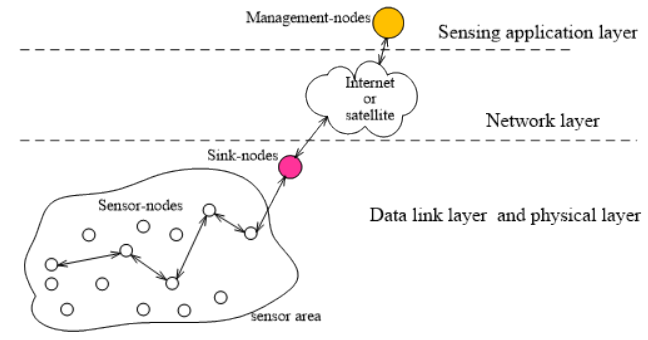

Fig. 1. WSN hierarchical structure based on TCP/IP protocol

\section{B. OpenFlow/SDN Architecture}

OpenFlow protocols are the core technology in SDN [6]. The two most critical parts are the OpenFlow switches and controllers. See fig.2.

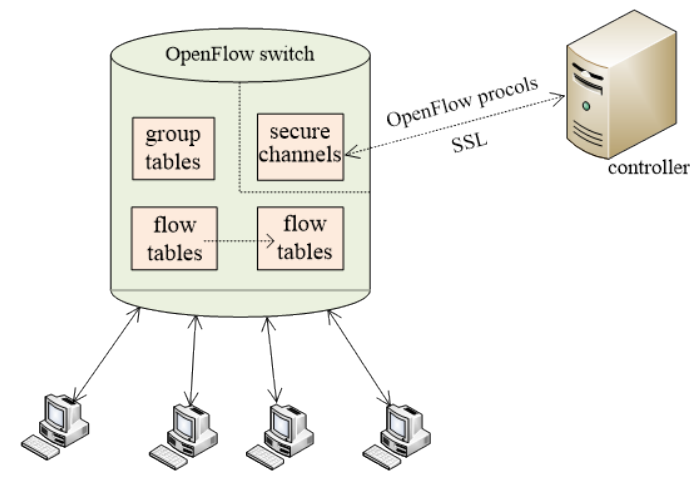

Fig. 2. OpenFlow architecture

OpenFlow switches mainly forward data. Controllers are based on the software platform, master the whole network topology and links' information [7]. All the packet matching and forwarding rules for the OpenFlow switches are programmed by controllers to manage uniformly network and develop the forward behaviors of OpenFlow switches according to the routing strategy. Flow tables are the core part in an OpenFlow switch composed of the flow table items and the actions that should be performed when the groupings that match the flow arrives. Whenever SDN devices receive a packet, they access the flow tables in a search matching flow table items. If SDN devices find a match, then a pre-configured action will be taken along with requiring the forward grouping. If not, the grouping will be discarded or the packet will be passed to the controllers. Group tables are responsible for looking up and forwarding the packets. Secure channels are used to connect to OpenFlow switches and controllers, so that data, configuration information and instructions among them can be transmitted securely based on SSL.

SDN is developed from OpenFlow which makes the control plane and data plane separated. Switches give some heavy control strategies to the controllers. Each of them runs independently, and the information forward strategy centrally published by controllers to the exchange devices. See the fig.3.

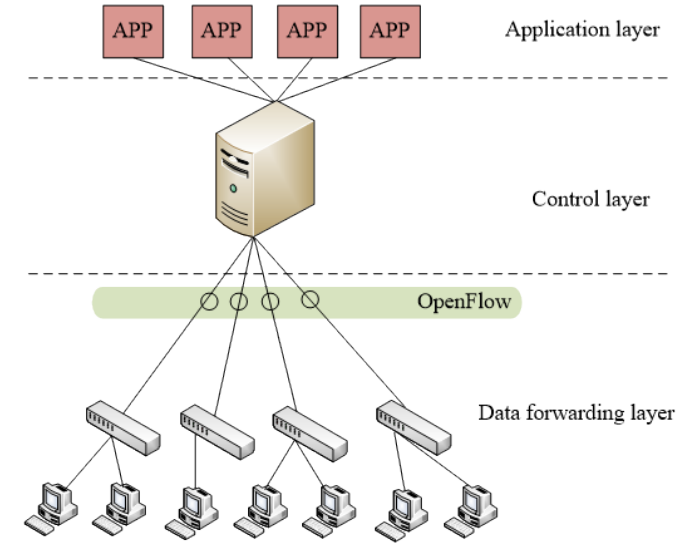

Fig. 3. Three layer architecture in SDN

In order to avoid frequent interactions, the rules are based on the flow. Controllers are usually implemented by network operating system (NOS). The application layer can flexibly program the network resource concerned by providing the global or local network abstract view which is provided by the control layer and control network flow flexibly. SDN abstracts the infrastructure network, which enables application software complete tasks without understanding the individual characteristics and styles of network devices. This level of abstraction also provides the ability to network virtualization and decoupling the network service from the underlying physical network.

\section{SD-SDN ARCHITECTURE MODEL}

The function of switching equipment based on TCP/IP network is still highly dependent on the closed hardware characteristics. The existing computer network architecture is difficult to meet WSN's development needs. OpenFlow technology known as standardized and compatible network virtualization technologies, has led to many innovative solutions in this field. In the literature [8], apply the OpenFlow technology to WSN and propose a new architecture model for WSN, which defines three roles as master nodes, center nodes and common nodes and adopts hybrid routing mechanism to improve the transmission efficiency to WSN [8]. However, WSN has limited energy and computing capacity. Information fusion can provide more accurate information; meanwhile, it reduces the nodes' calculation and transmission time. Therefore, in this article, modify the model proposed in literature [8], then introduce the OpenFlow/SDN technology to WSN architecture and add letter melt layer. Establish software defined wireless sensor networks (SD-WSN) hierarchical architecture model.

\section{A. SD-WSN hierarchical architecture}

OpenFlow/SDN technology is used in SD-WSN to reorganize and divide the architecture hierarchy of WSN. Besides, set management nodes as service combining with OpenFlow to realize the separation of logic control and network forwarding equipments in WSN. Take sink nodes as data forwarding layer equivalent to the OpenFlow switches in SDN. See fig.4. 


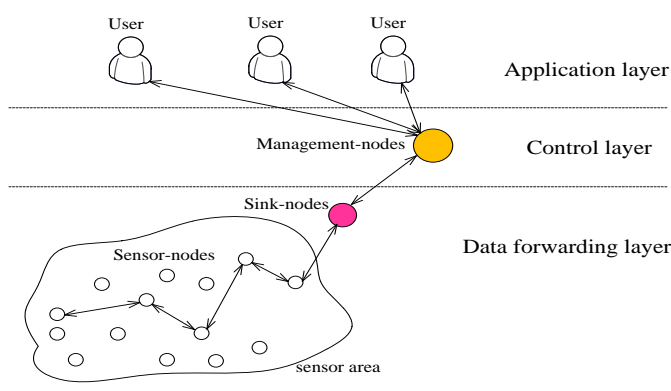

Fig. 4. Three layer architecture in SD-WSN

In SDN, the data forwarding is completed by the OpenFlow switches; controllers pass some OpenFlow protocol instructions to the OpenFlow switches through secure channels. This requires that sink nodes have ability to match flow table items automatically, and identify various existing WSN routing protocols to match and forward the data flow in WSN, meanwhile, the deployment extension programs are passed to each management nodes through the reservation interfaces to realize the flexible control over the entire network, and then control the overall flow and QoS in SD-WSN. The sensor nodes are only responsible for receiving and executing the data flow. From this view, in SD-WSN, sink nodes are only operational entities; management nodes are the control center equivalent to the SDN controller, they can provide a programmable network operating platform for the OpenFlow network architecture.

In the actual WSN network application, information fusion technology can automatically carry out correlation, statistics, analysis and synthesis to perceptual data under certain guidelines, and perform evaluation tasks accurately. FlowVisor uses network virtualization technology to establish a network virtual layer between the OpenFlow switches and controllers [8].

This process combines the underlying network data information into logical slices, users can seamlessly control and orchestrate allocated virtual resource for efficient delivery of network service by it. See fig.5.

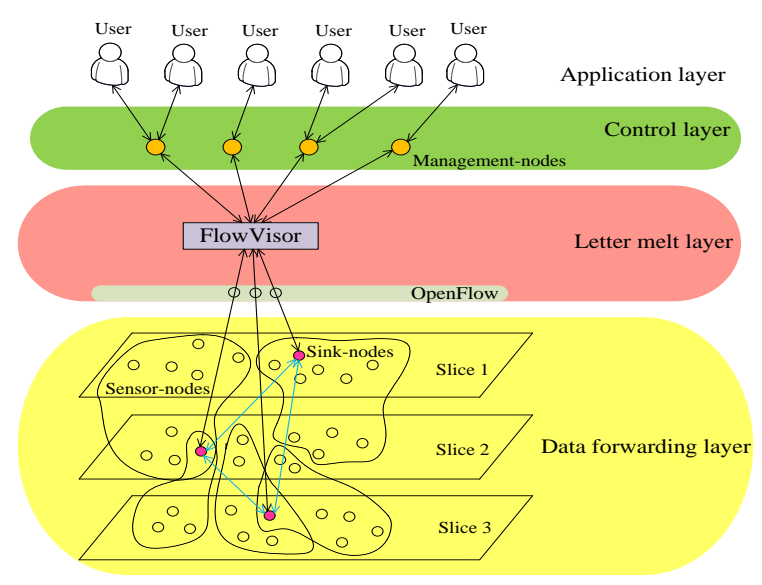

Fig. 5. Four layer architecture in SD-WSN

FlowVisor uses the standard OpenFlow instruction set to manage sink nodes and generates independent network slices by dividing flow tables' space [9]. Virtual network topology is determined by the current flow in the network. Instantiation creates a set of applications for the virtual network on the controllers only; users' experiments on each piece do not interfere with each other. FlowVisor controllers are introduced into SD-WSN to abstract the underlying network, links and topology into virtualization layer, and fuse into smaller data flow for multi-sensor data information in network. Sink nodes forward to the next hop with a more determined exit. Sensor nodes only provide sensory perception tasks, which can be used to integrate sensory data and their own historical data. So the letter melt layer' integration enhances the controllers' control ability and efficiency.

\section{B. SD-WSN information forwarding mechanism}

The purpose of rebuilding WSN architecture model is able to maximize perceived objects' characteristics, classify and fuse the nodes' data to forward resource fully, so as to improve the stability and transmission efficiency of the network system. Therefore, nodes' information forwarding strategy is especially critical.

The core idea of the SD-WSN model designed in this article is that all the control parts and the forward parts are extracted, managed by management nodes. Establish or alter transmission paths according to the information QoS and network slices. When sink nodes receive the forward request, they check the flow tables to see whether the flow table items exist. If the information flow can match existing flow table items, it can be forwarded based on the forwarding strategy that has been built. If the information is received for the first time, sink nodes will match flow tables' information and feedback to the management nodes to request to build the forwarding paths, management nodes will look up the optimal forwarding paths according to the network state, nodes’ performance and QoS after receiving the management nodes' feedback information and build anti-paths to them. Create flow tables in the sink nodes, thus, when the same forwarding requests reach the sink nodes again, there is no need to rebuild forwarding paths. They can be forwarded by the forwarding strategies recorded in the existing flow tables.

Management nodes completely control the forwarding paths. They can adjust forwarding paths whenever link failures, channel interference or node energy exhaustion. Therefore, the performance of SD-WSN is better than the traditional WSN.

\section{Simulation AND EVALUATION}

This section evaluates SD-WSN architecture model, then compares and analyzes the traditional WSN routing protocols. The experiment parameters are as table1:

TABLE I. SIMULATION PARAMETERS

\begin{tabular}{|c|c|}
\hline parameters & value \\
\hline$E_{0}$ & $0.5 \mathrm{~J}$ \\
\hline$k$ & $4000 \mathrm{bit}$ \\
\hline$E_{\mathrm{fs}}$ & $10 \mathrm{pJ} / \mathrm{bit} / \mathrm{m}^{2}$ \\
\hline$E_{m p}$ & $0.0013 \mathrm{pJ} / \mathrm{bit} / \mathrm{m}^{4}$ \\
\hline
\end{tabular}


The range of the experiment site is $100 \mathrm{~m} * 100 \mathrm{~m}$. 100 nodes are randomly deployed in the network. As the running times increasing, the changes in the number of surviving nodes in the network are shown in fig.6. Compare to the CAMAC protocol and the LEACH protocol, the CAMAC protocol starts to have some nodes dead when running to about 260 times, the LEACH protocol starts to have some nodes dead when running to about 600 times. While the new architecture (NEW) proposed in this article starts to die when running to about 1200 times, by this time, nodes in the CAMAC protocol and the LEACH protocol has been exhausted and died completely. Therefore, the new architecture extends network lift cycle, satisfies QoS requirements on multi-information business, and reduces network cost and energy consumption.

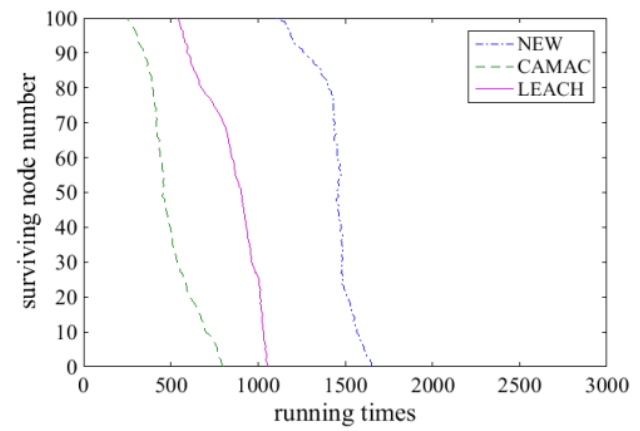

Fig. 6. Change of surviving nodes

SD-WSN can choose the optimal transmission path by the state and change of network topology. See fig.7. The data transmission in NEW has been the highest compared to the traditional WSN routing protocols with the running times increasing. It is at least 5 times higher than the LEACH protocol and 2 times higher than the CAMAC protocol. The new architecture is still optimal in terms of network transmission efficiency.

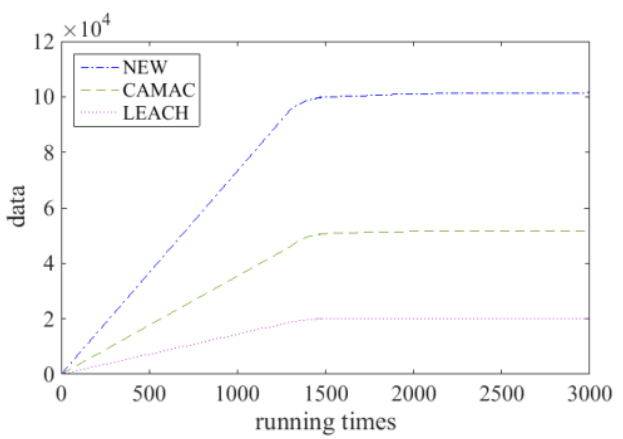

Fig. 7. Transmission efficiency

\section{CONCLUSION}

In order to adapt the traditional WSN to the development of Internet emerging technology, improve WSN architecture and technical indicators, this article proposes SD-WSN hierarchy model by combining SDN and WSN with OpenFlow technology. Using network virtual technology to integrate the data acquisition and forwarding to underlying nodes' data. Refine the SD-WSN hierarchy and add letter melt layer between the control layer and the forwarding layer to further improve the network information forwarding strategy. Simulate the new architecture model and compare to the traditional WSN routing protocols, the results show that the new architecture can extend network life cycle, and the data transmission efficiency in network is improved obviously.

\section{REFERENCES}

[1] P. Huang, L. Xiao, S. Soltani, M.W. Mutka, N. Xi, "The evolution of MAC protocols in wireless sensor networks: a survey," IEEE Communications Surveys and Tutorials, America, vol.15, pp. 101-120, May 2013.

[2] D. Zhang, J. Guo, C.M. Wu, "Controller placement based on hierarchical multi-center SDN,” Acta Electronica Sinica, vol.45, pp.680686, March 2017. (in Chinese)

[3] J. Chen, K. He, R. Du, M. Zhang, Y. Xiang, et al. "Dominating set and network coding-based routing in wireless mesh networks," IEEE Transactions on Parallel \& Distributed Systems, America, vol.26, pp.423-433, Feburay 2015.

[4] J. Domingues, A. Damaso, N. Rosa, "Smart: service model for integration wireless sensor networks and the Internet," IEEE International Conference on Parallel \& Distributed Systems, Washington, January 2011.

[5] H. Zhao, S.L. Shao, J. Zhu, K. Zhang, "Design and implementation of a multi-core embedded gateway for linking WSN and Internet,” Journal of Northeastern University (Natural Science), Shenyang, vol.33, pp.65-68, January 2012. (in Chinese)

[6] N. Mckeown, T. Anderson, H. Balakrishnan, G. Parulkar, L. Peterson, et al, "OpenFlow: enabling innovation in campus networks," Acm Sigcomm Computer Communication Review, America, vol.38, pp.69-74 April 2008.

[7] M.B, Aleksander, "Implementation technology software defined networking in wireless sensor networks,” 2015 IEEE 8th International Conference on Intelligent Data Acquisition and Advanced Computing Systems: Technology and Applications, Warsaw, vol.1, pp.448-452, September 2015.

[8] Z.J. Han, W.L. Ren, “A novel wireless sensor networks structure based on the SDN," International Journal of Distributed sensor networks, America, vol.10, pp.1-7, January 2014.

[9] R. Sherwood, M. Gibb, K.K. Yan, "FlowVisor: a network virtualization layer,” OpenFlow-tr-2009-1, Palo Alto, California: Stanford University, October 2009. 